\title{
Formulation of Hamiltonian equations for fractional variational problems
}

September 3, 2018

\author{
Sami I. Muslih ${ }^{1}$ \\ Department of Physics, Al-Azhar University, Gaza, Palestine \\ and \\ International Center for Theoretical Physics(ICTP), \\ Trieste, Italy
}

Dumitru Baleanu ${ }^{2}$

Department of Mathematics and Computer Sciences, Faculty of Arts and Sciences, Çankaya University- 06530, Ankara, Turkey

\begin{abstract}
An extension of Riewe's fractional Hamiltonian formulation is presented for fractional constrained systems. The conditions of consistency of the set of constraints with equations of motion are investigated. Three examples of fractional constrained systems are analyzed in details.

PACS: 02.30-f, 11.10.Ef

Key words: fractional derivative, Hamiltonian system, Non-conservative systems.

\footnotetext{
${ }^{1}$ E-mail: smuslih@ictp.trieste.it

${ }^{2}$ On leave of absence from Institute of Space Sciences, P.O.BOX, MG-23, R 76900, Magurele-Bucharest, Romania,E-mails: dumitru@cankaya.edu.tr, baleanu@venus.nipne.ro
} 


\section{Introduction}

Fractional derivatives [1-6], have played a significant role in engineering, science, and pure and applied mathematics during the last decade [7-10]. Fractional calculus found many applications in recent studies of scaling phenomena [11-13]. One can find other important and interesting application of fractional calculus in classical mechanics [14-20]. Riewe has used the fractional calculus to develop a formalism which can be used for both conservative and non conservative systems $[14,15]$. Although many laws of nature can be obtained using certain functionals and the theory of calculus of variations, not all laws can be obtained by this way. For example, almost all systems contain internal damping, yet traditional energy based approach cannot be used to obtain equations describing the behavior of a non conservative system $[14,15]$.By this approach, one can obtain the Lagrangian and the Hamiltonian equations of motion for the nonconservative systems .

Klimek have introduced the symmetric fractional derivative and the EulerLagrange equations for models depending on sequential derivatives were obtained by using the minimal action principle [9]. In addition, the corresponding Hamiltonian was introduced and the Hamilton's equations were analyzed [9]. The models described by fractional order derivatives of RiemannLiouville type in sequential form were analyzed in both Lagrangian and Hamiltonian formalisms in [10].

Taking into account the constrained systems play an important role in gauge theories and the distinguishing feature of singular Lagrangian theories in the Hamiltonian formulation is the presence of constraints, we believe that the application of fractional calculus to constrained systems presents interest both from theoretical and practical point of views. Recently, an extension of the simplest fractional problem and the fractional variational problem of Lagrange was obtained [18,19]. A natural generalization of Agrawal's approach $[18,19]$, is to apply the fractional calculus to constrained systems [21-22] and to obtain the Hamiltonian formulation of constrained systems.

The main aim of this paper is to obtain the Hamiltonian equations of motion for fractional variational problems with constraints.

The plan of this paper is as follows:

In Sect. 2 Riemnann-Liouville fractional derivatives are briefly reviewed. Sect. 3 is dealing with fractional Hamiltonian equations initiated by Riewe. Sect. 4 presents the Agrawal's approach of the fractional Euler-Lagrange equations. In Sect. 5 the extension of Riewe's formulation for the constrained 
systems is obtained. Three examples of fractional constrained systems are investigated in Sect. 6 . Finally, Sect. 7 is dedicated to our conclusions.

\section{Riemann-Liouville fractional derivative}

Several definitions of a fractional derivative has been proposed. These definitions include Riemann-Liouville, Grunwald-Letnikov, Weyl, Caputo, Marchaud and Riesz fractional derivatives [1-6]. Here, we formulate the problem in terms of the left and right Riemann-Liouville fractional derivatives, which are defined as [5]

The left Riemann - Liouville fractional derivative

$$
{ }_{a} \mathbf{D}_{t}^{\alpha} f(t)=\frac{1}{\Gamma(n-\alpha)}\left(\frac{d}{d t}\right)^{n} \int_{a}^{t}(t-\tau)^{n-\alpha-1} f(\tau) d \tau,
$$

and

The right Riemann - Liouville fractional derivative

$$
{ }_{t} \mathbf{D}_{b}^{\alpha} f(t)=\frac{1}{\Gamma(n-\alpha)}\left(-\frac{d}{d t}\right)^{n} \int_{t}^{b}(\tau-t)^{n-\alpha-1} f(\tau) d \tau,
$$

where the order $\alpha$ fulfills $n-1 \leq \alpha<n$ and $\Gamma$ represents the Euler's gamma

function. If $\alpha$ is an integer, these derivatives are defined in the usual sense, i.e.,

$$
{ }_{a} \mathbf{D}_{t}^{\alpha} f(t)=\left(\frac{d}{d t}\right)^{\alpha}, \quad{ }_{t} \mathbf{D}_{b}^{\alpha} f(t)=\left(-\frac{d}{d t}\right)^{\alpha}, \alpha=1,2, \ldots
$$

\section{Riewe's formulation}

Now we shall briefly review Riewe's formulation of fractional generalization of Lagrangian and Hamiltonian equations of motion.

Let us consider the action function of the form $[14,15]$

$$
S=\int_{a}^{b} L\left(\left\{q_{n}^{r}, Q_{n^{\prime}}^{r}\right\}, t\right) d t
$$

where the generalized coordinates are defined as follows:

$$
\left.\left.q_{n}^{r}:={ }_{a} \mathbf{D}_{t}^{\alpha}\right)^{n} x_{r}(t), Q_{n^{\prime}}^{r}:={ }_{t} \mathbf{D}_{b}^{\alpha}\right)^{n^{\prime}} x_{r}(t),
$$


and $r=1,2, \ldots, R$ denotes the number of fundamental coordinates, $n=$ $0, \ldots, N$, the sequential order of the derivatives defining the generalized coordinates $q$, and $n^{\prime}=1, \ldots, N^{\prime}$ the sequential order of the derivatives in definition of the coordinates $Q$. A necessary condition for $S$ to admit an extremum for given functions $x_{r}(t)$ is that $x_{r}(t)$ satisfy the Euler-Lagrange equations $[14,15]$

$$
\frac{\partial L}{\partial q_{0}^{r}}+\sum_{n=1}^{N}\left({ }_{t} \mathbf{D}_{b}^{\alpha}\right)^{n} \frac{\partial L}{\partial q_{n}^{r}}+\sum_{n^{\prime}=1}^{N^{\prime}}\left({ }_{a} \mathbf{D}_{t}^{\alpha}\right)^{n^{\prime}} \frac{\partial L}{\partial Q_{n^{\prime}}^{r}}=0 .
$$

Following references $[14,15]$, the generalized momenta take the form

$$
\begin{aligned}
& p_{n}^{r}=\sum_{k=n+1}^{N}\left({ }_{t} \mathbf{D}_{b}^{\alpha}\right)^{k-n-1} \frac{\partial L}{\partial q_{k}^{r}}, \\
& \pi_{n^{\prime}}^{r}=\sum_{k=n^{\prime}+1}^{N^{\prime}}\left({ }_{a} \mathbf{D}_{t}^{\alpha}\right)^{k-n^{\prime}-1} \frac{\partial L}{\partial Q_{k}^{r}} .
\end{aligned}
$$

The canonical Hamiltonian has the following form:

$$
H=\sum_{r=1}^{R} \sum_{n=0}^{N-1} p_{n}^{r} q_{n+1}^{r}+\sum_{r=1}^{R} \sum_{n^{\prime}=0}^{N^{\prime}-1} \pi_{n^{\prime}}^{r} Q_{n^{\prime}+1}^{r}-L .
$$

The Hamilton's equations of motion read as

$$
\frac{\partial H}{\partial q_{N}^{r}}=0, \frac{\partial H}{\partial Q_{N^{\prime}}^{r}}=0
$$

For $n=1, \ldots, N, n^{\prime}=1, \ldots, N^{\prime}$ we have the following equations of motion

$$
\begin{aligned}
& \frac{\partial H}{\partial q_{n}^{r}}={ }_{t} \mathbf{D}_{b}^{\alpha} p_{n}^{r}, \quad \frac{\partial H}{\partial Q_{n^{\prime}}^{r}}={ }_{a} \mathbf{D}_{t}^{\alpha} \pi_{n^{\prime}}^{r}, \\
& \frac{\partial H}{\partial q_{0}^{r}}=-\frac{\partial L}{\partial q_{0}^{r}}={ }_{t} \mathbf{D}_{b}^{\alpha} p_{0}^{r}+{ }_{a} \mathbf{D}_{t}^{\alpha} \pi_{0}^{r} .
\end{aligned}
$$

The other equations are given by

$$
\begin{aligned}
& \frac{\partial H}{\partial p_{n}^{r}}=q_{n+1}^{r}={ }_{a} \mathbf{D}_{t}^{\alpha} q_{n}^{r}, \quad \frac{\partial H}{\partial \pi_{n^{\prime}}^{r}}=Q_{n+1}^{r}={ }_{t} \mathbf{D}_{b}^{\alpha} Q_{n^{\prime}}^{r}, \\
& \frac{\partial H}{\partial t}=-\frac{\partial L}{\partial t},
\end{aligned}
$$

where, $n=0, \ldots, N, n^{\prime}=1, \ldots, N^{\prime}$. 


\section{Agrawal's approach}

Agrawal have obtained the Euler-Lagrange equations for fractional variational problems [18]. Now we would like to give review for his approach.

Consider the action function

$$
S\left[q_{0}^{1}, \ldots q_{0}^{R}\right]=\int_{a}^{b} L\left(\left\{q_{n}^{r}, Q_{n^{\prime}}^{r}\right\}, t\right) d t
$$

subject to the independent constraints

$$
\Phi_{m}\left(t, q_{0}^{1}, \ldots, q_{0}^{R}, q_{n}^{r}, Q_{n^{\prime}}^{r}\right)=0, m<R,
$$

where the generalized coordinates are defined as follows:

$$
\left.q_{n}^{r}:=\left({ }_{a} \mathbf{D}_{t}^{\alpha}\right)^{n} x_{r}(t), Q_{n^{\prime}}^{r}:={ }_{t} \mathbf{D}_{b}^{\beta}\right)^{n^{\prime}} x_{r}(t),
$$

Then the necessary condition for the curves $q_{0}^{1}, \ldots, q_{0}^{R}$ with the boundary conditions $q_{0}^{r}(a)=q_{0}^{r a}, q_{0}^{r}(b)=q_{0}^{r b}, r=1,2,, \ldots, R$, to be an extremal of the

functional given by equation (14) is that the functions $q_{0}^{r}$ satisfy the following Euler-Lagrange equations [18]:

$$
\frac{\partial \bar{L}}{\partial q_{0}^{r}}+\sum_{n=1}^{N}\left({ }_{t} \mathbf{D}_{b}^{\alpha}\right)^{n} \frac{\partial \bar{L}}{\partial q_{n}^{r}}+\sum_{n^{\prime}=1}^{N^{\prime}}\left({ }_{a} \mathbf{D}_{t}^{\alpha}\right)^{n^{\prime}} \frac{\partial \bar{L}}{\partial Q_{n^{\prime}}^{r}}=0,
$$

where $\bar{L}$ is defined as [18]

$$
\bar{L}\left(\left\{q_{n}^{r}, Q_{n^{\prime}}^{r}\right\}, t, \lambda_{m}(t)\right)=L\left(\left\{q_{n}^{r}, Q_{n^{\prime}}^{r}\right\}, t\right)+\lambda_{m}(t) \Phi_{m}\left(t, q_{0}^{1}, \ldots, q_{0}^{R}, q_{n}^{r}, Q_{n^{\prime}}^{r}\right),
$$

here, the multiple $\lambda_{m}(t) \in R^{m}$ are continuous on $[a, b]$.

\section{Fractional Hamiltonian formulation}

In fractional calculus it is not a unique way to define the Hailtonian for a given Lagrangian, mainly because they are several definitions of fractional derivatives.

To obtain the Hamilton's equations for the the fractional variational problems, we need to re-define the lift and the right canonical momenta as follows:

$$
\begin{aligned}
& p_{n}^{r}=\sum_{k=n+1}^{N}\left({ }_{t} \mathbf{D}_{b}^{\alpha}\right)^{k-n-1} \frac{\partial \bar{L}}{\partial q_{k}^{r}}, \\
& \pi_{n^{\prime}}^{r}=\sum_{k=n^{\prime}+1}^{N^{\prime}}\left({ }_{a} \mathbf{D}_{t}^{\alpha}\right)^{k-n^{\prime}-1} \frac{\partial \bar{L}}{\partial Q_{k}^{r}} .
\end{aligned}
$$


The canonical Hamiltonian has the following form:

$$
\bar{H}=\sum_{r=1}^{R} \sum_{n=0}^{N-1} p_{n}^{r} q_{n+1}^{r}+\sum_{r=1}^{R} \sum_{n^{\prime}=0}^{N^{\prime}-1} \pi_{n^{\prime}}^{r} Q_{n^{\prime}+1}^{r}-\bar{L} .
$$

Then, the modified canonical equations of motion are obtained as

$$
\begin{aligned}
& \left\{q_{n}^{r}, \bar{H}\right\}={ }_{t} \mathbf{D}_{b}^{\alpha} p_{n}^{r},\left\{Q_{n^{\prime}}^{r}, \bar{H}\right\}={ }_{a} \mathbf{D}_{t}^{\alpha} \pi_{n^{\prime}}^{r}, \\
& \left\{q_{0}^{r}, \bar{H}\right\}={ }_{t} \mathbf{D}_{b}^{\alpha} p_{0}^{r}+{ }_{a} \mathbf{D}_{t}^{\alpha} \pi_{0}^{r} .
\end{aligned}
$$

Here, $n=1, \ldots, N, n^{\prime}=1, \ldots, N^{\prime}$.

The other set of equations of motion are obtained as

$$
\begin{aligned}
& \left.\left\{p_{n}^{r}, \bar{H}\right\}=q_{n+1}^{r}={ }_{a} \mathbf{D}_{t}^{\alpha} q_{n}^{r},\left\{\pi_{n^{\prime}}^{r}, \bar{H}\right\}\right\}=Q_{n+1}^{r}={ }_{t} \mathbf{D}_{b}^{\alpha} Q_{n^{\prime}}^{r}, \\
& \frac{\partial \bar{H}}{\partial t}=-\frac{\partial \bar{L}}{\partial t} .
\end{aligned}
$$

Here, $n=0, \ldots, N, n^{\prime}=1, \ldots, N^{\prime}$ and the commutator $\{$,$\} is the Poisson's$ bracket and it is defined as

$$
\{A, B\}_{q_{n}^{r}, p_{n}^{r}, Q_{n^{\prime}}^{r}, \pi_{n^{\prime}}^{r}}=\frac{\partial A}{\partial q_{n}^{r}} \frac{\partial B}{\partial p_{n}^{r}}-\frac{\partial B}{\partial q_{n}^{r}} \frac{\partial A}{\partial p_{n}^{r}}+\frac{\partial A}{\partial Q_{n^{\prime}}^{r}} \frac{\partial B}{\partial \pi_{n^{\prime}}^{r}}-\frac{\partial B}{\partial Q_{n^{\prime}}^{r}} \frac{\partial A}{\partial \pi_{n^{\prime}}^{r}},
$$

where, $n=0, \ldots, N, n^{\prime}=1, \ldots, N^{\prime}$.

\section{Examples}

In this section, we obtain the Hamiltonian equations for an constrained fractional variational problems. All calculations were done for $0<\alpha<1$.

\subsection{Example 1}

As the first example, consider the following constrained fractional variational problem from the optimal control theory [18]: minimize

$$
S\left[x_{1}, x_{2}\right]=\frac{1}{2} \int_{0}^{1}\left[x_{1}^{2}+x_{2}^{2}\right] d t
$$

such that

$$
\begin{aligned}
& { }_{0} \mathbf{D}_{t}^{\alpha} x_{1}=-x_{1}+x_{2}, \\
& x_{1}(0)=1
\end{aligned}
$$


The modified Lagrangian $\bar{L}$ is given by

$$
\bar{L}=\frac{1}{2}\left[x_{1}^{2}+x_{2}^{2}\right]+l \phi_{1}+\lambda \phi_{2},
$$

where $l, \lambda$ are Lagrange multipliers and $\phi_{1}, \phi_{2}$ are the constraints and are defined as

$$
\begin{aligned}
& \phi_{1}={ }_{0} \mathbf{D}_{t}^{\alpha} x_{1}+x_{1}-x_{2}=0, \\
& \phi_{2}=x_{1}(0)-1=0 .
\end{aligned}
$$

The generalized canonical momenta read as

$$
p_{0}^{1}=l, \quad p_{0}^{2}=0 .
$$

The Hamiltonian $\bar{H}$ is given by

$$
\bar{H}=p_{0}^{1} q_{1}^{1}-\frac{1}{2}\left[x_{1}^{2}+x_{2}^{2}\right]-l \phi_{1}-\lambda \phi_{2},
$$

The Hamiltonian equations are obtained as

$$
\begin{gathered}
\frac{\partial \bar{H}}{\partial q_{1}^{1}}=p_{0}^{1}-l=0, \\
\frac{\partial \bar{H}}{\partial q_{1}^{2}}=0,
\end{gathered}
$$

which is identically satisfied. The other equations of motion are given by

$$
\begin{aligned}
& \frac{\partial \bar{H}}{\partial x_{1}}=-x_{1}-l={ }_{t} \mathbf{D}_{1}^{\alpha}\left(p_{0}^{1}\right), \\
& \frac{\partial \bar{H}}{\partial x_{2}}=-x_{2}+l=0 .
\end{aligned}
$$

Making use of (33), equations (36) and (37) can be put in the form,

$$
\begin{aligned}
& x_{1}+l+{ }_{t} \mathbf{D}_{1}^{\alpha}(l)=0, \\
& x_{2}-l=0 .
\end{aligned}
$$

Equations (38) and (39) are in exact agreement with those obtained by using the Euler-Lagrange formulation for fractional variational problems [18]. 


\subsection{Example 2}

Let us consider the following Lagrangian

$$
L=\frac{1}{2}\left(\dot{x}_{1}+\dot{x}_{2}\right)^{2} .
$$

The Lagrangian (40) is singular because its usual Hessian matrix has rank 1. One possible fractional generalization of (40) is given as follows

$$
\bar{L}=\frac{1}{2}\left({ }_{0} \mathbf{D}_{t}^{\alpha} x_{1}+{ }_{0} \mathbf{D}_{t}^{\alpha} x_{2}\right)^{2} .
$$

For Lagrangian (41) we introduce the notion of fractional Hessian as

$$
\frac{\partial^{2} \bar{L}}{\partial_{0} \mathbf{D}_{t}^{\alpha} x_{i 0} \mathbf{D}_{t}^{\alpha} x_{j}}, i, j=1,2 .
$$

Evaluating the rank of (51) we obtain the result being 1, so the system is a constrained system.

On the other hand from (41) we obtain immediately the form of the canonical momenta as

$$
p_{0}^{1}={ }_{0} \mathbf{D}_{t}^{\alpha} x_{1}+{ }_{0} \mathbf{D}_{t}^{\alpha} x_{2}, p_{0}^{2}={ }_{0} \mathbf{D}_{t}^{\alpha} x_{1}+{ }_{0} \mathbf{D}_{t}^{\alpha} x_{2},
$$

so that (43) implies that

$$
p_{0}^{2}-p_{0}^{1}=0
$$

which represents a primary constraint.

Using (41) and (43) the form of the canonical fractional Hamiltonian is given by

$$
\bar{H}=\frac{1}{2}\left(p_{0}^{1}\right)^{2}+{ }_{0} \mathbf{D}_{t}^{\alpha} x_{2}\left(p_{0}^{2}-p_{0}^{1}\right) .
$$

If we denote in (45) ${ }_{0} \mathbf{D}_{t}^{\alpha} x_{2}$ by $\lambda$ we observe that canonical fractional Hamiltonian becomes

$$
\bar{H}=\frac{1}{2}\left(p_{0}^{1}\right)^{2}+\lambda\left(p_{0}^{2}-p_{0}^{1}\right)
$$

From (46) we obtain the fractional Hamiltonian motions as follows

$$
{ }_{0} \mathbf{D}_{t}^{\alpha} x_{1}=p_{0}^{1}-\lambda,{ }_{0} \mathbf{D}_{t}^{\alpha} x_{2}=\lambda
$$




$$
{ }_{0} \mathbf{D}_{t}^{\alpha} p_{0}^{1}=0,{ }_{0} \mathbf{D}_{t}^{\alpha} p_{0}^{2}=0 .
$$

Using (44) and (48) we conclude that the primary surface of constraints is preserved after considering its evolution from the fractional calculus point of view.

\subsection{Example 3}

Finally, let us analyze the following Lagrangian

$$
L=\frac{1}{2}\left(\dot{x}_{1}\right)^{2}-\dot{x}_{2} x_{3}
$$

One possible generalization of (49) is given as

$$
\bar{L}=\frac{1}{2}\left({ }_{0} \mathbf{D}_{t}^{\alpha} x_{1}\right)^{2}-\left({ }_{0} \mathbf{D}_{t}^{\alpha} x_{2}\right) x_{3} .
$$

The fractional Hessian corresponding to (50) is given by

$$
\frac{\partial^{2} \bar{L}}{\partial_{0} \mathbf{D}_{t}^{\alpha} x_{i 0} \mathbf{D}_{t}^{\alpha} x_{j}}, i, j=1,3 .
$$

has rank one, therefore the fractional Lagrangian (150) is degenerate admitting two primary constraints.

Using (50) we obtain the momenta as follows

$$
p_{0}^{1}={ }_{0} \mathbf{D}_{t}^{\alpha} x_{1}, p_{0}^{2}=x_{3}, p_{0}^{3}=0 .
$$

From (52) we identify the primary constraints as

$$
p_{0}^{2}-x_{3}=0, p_{0}^{3}=0 .
$$

The canonical Hamiltonian in this case is given by

$$
\bar{H}=\frac{\left(p_{0}^{1}\right)^{2}}{2}+{ }_{0} \mathbf{D}_{t}^{\alpha} x_{2}\left(p_{0}^{2}-x_{3}\right) .
$$

Denoting ${ }_{0} \mathbf{D}_{t}^{\alpha} x_{2}$ by $\lambda$ and by using (154) the canonical equations become

$$
\begin{aligned}
& { }_{0} \mathbf{D}_{t}^{\alpha} x_{1}=p_{0}^{1},{ }_{0} \mathbf{D}_{t}^{\alpha} x_{3}=0,{ }_{0} \mathbf{D}_{t}^{\alpha} x_{2}=\lambda, \\
& { }_{0} \mathbf{D}_{t}^{\alpha} p_{0}^{1}=0,{ }_{0} \mathbf{D}_{t}^{\alpha} p_{0}^{3}=-\lambda,{ }_{0} \mathbf{D}_{t}^{\alpha} p_{0}^{2}=0, p_{0}^{2}-q_{3}=0 .
\end{aligned}
$$


We observed from (55) that the consistency conditions of the primary constraint $p_{0}^{2}-x_{3}=0$ is compatible with the equations of motion.

The evolution of the second primary constraint makes $\lambda$ zero, therefore

$$
{ }_{0} \mathbf{D}_{t}^{\alpha} x_{2}=0 \text {. }
$$

From (56) we conclude that a new secondary constraint appears, therefore the initial phase-space was modified.

\section{Conclusion}

The fractional quantization problem is an open and important issue. The main difficulty is related to the non-locality of fractional Lagrangian. Another specific problem in this field is related to the existence of various definitions of fractional derivatives which lead to different forms of fractional Lagrangians and fractional Hamiltonians.

In this study, the Hamiltonian equations have been obtained for unconstrained and constrained variational problem, in the same manner as those obtained by using the formulation of Euler-Lagrange equations for variational problems obtained in [18]. The Hamiltonian describes a non-conservative system, therefore the the Poisson brackets introduced in this study leads us to a Hamiltonian conserved in time in the limit $\alpha \rightarrow 1$ and for Hamiltonian which does not explicitly depends on time. In this paper two different systems with constraints were investigated, the first kind corresponds to a fractional Lagrangian containing Lagrange multipliers. For this case the fractional Hamiltonian and its corresponding equations were obtained.

The second kind of constrained system involves primary and secondary constraints. Having in mind to obtain similar results with the usual analysis of constrained systems in the limit process we investigated the form of the equations of motion and the evolution of the surface of primary constraints for the last two examples. It was observed that the form of the primary constraints and the form of the Hamiltonian equations are in agreement with each other for the second example but for the third one the consistency conditions impose new constraints.

In the general case the primary constraints may produce secondary constraints and the process may continue until no new constraints will appear. 


\section{Acknowledgments}

S. M. would like to thank the Abdus Salam International Center for Theoretical Physics, Trieste, Italy, for support and hospitality during the preparation

of this work. This work was done within the framework of the Associateship Scheme of the Abdus Salam ICTP.

D. B. would like to thank O. Agrawal and J. A. Tenreiro Machado for interesting discussions and to K. B. Oldham for encouragements. This work is partially supported by the Scientific and Technical Research Council of Turkey.

\section{References}

[1] K. S. Miller and B. Ross: An Introduction to the Fractional Integrals and Derivatives-Theory and Applications, Gordon and Breach, Longhorne, PA, (1993).

[2] S. G. Samko, A. A. Kilbas and O. I. Marichev: Fractional Integrals and Derivatives- Theory and Applications, Gordon and Breach, Linghorne, PA, (1993).

[3] K. B. Oldham and J. Spanier: The Fractional Calculus, Academic Press, New York, (1974).

[4] R. Gorenflo and F. Mainardi: Fractional calculus: Integral and Differential Equations of Fractional Orders, Fractals and Fractional Calculus in Continoum Mechanics, Springer Verlag, Wien and New York (1997).

[5] I. Podlubny: Fractional Differential Equations, Academic Press, New York, (1999).

[6] R. Hilfer: Applications of Fraction Calculus in Physics, World Scientific Publishing Company, Singapore, New Jersy, London and Hong Kong, (2000).

[7] J.A.Tenreiro Machado: Fract. Calc. Appl. Anal. 8(1) (2003)73-80.

J.A. Tenreiro Machado: Fract. Calc. Appl. Anal. 4(1)(2001) 47-68.

[8] O.P. Agrawal: Trans. ASME Journ. Vibrat. Acoust. (2004), to appear. 
[9] M. Klimek: Czech. J. Phys. 51 (2001)1348-1354; 52 (2002) 1247-1253.

[10] M. Klimek:Czech. J. Phys.52 (2002) 1247-1253.

[11] T. F. Nonnenmacher: J. Phys. A 23 (1990) L697S-L700S.

[12] R. Metzler and K. Joseph: Physica A. 278 (2000) 107-125 .

[13] G. M. Zaslavsky: Phys. Rep. 371(2002) 461-580.

[14] F. Riewe: Phys. Rev. E 53 (1996)1890-1899.

[15] F. Riewe: Phys. Rev. E 55 (1997)3581-3592.

[16] E. Rabei and T. Alhalholy: Int. J. Theor. Phys. A 19(17-18) 3083-3092.

[17] E. Rabei, T. Alhalholy and A. Tanni: Turk. J. Phys. 28 (2004) 1-9.

[18] O. P. Agrawal: J. Math. Anal. Appl. 272 (2002) 368-379.

[19] O.P. Agrawal: Trans. ASME, J. Math. Anal. Appl. 68 (2001) 339-341.

[20] O. P. Agrawal, J. Gregory and K. P. Spector: J. Math. Ann. Appl. 210 (1997) 702-711.

[21] D. Baleanu and T. Avkar: Nuovo Cimento 119 (2004) 73-79.

[22] D. Baleanu: Constrained systems and Riemann-Liouville fractional derivative, $\mathrm{n}$ Proceedings of $1^{\text {st }}$ IFAC Workshop on Fractional Differentiation and its Applications, Bordeaux, France, July 19-21 (2004) 597-602. 